\title{
O acervo de mosquitos (Diptera, Culicidae) de Nelson L. Cerqueira na Coleção de Invertebrados do Instituto Nacional de Pesquisas da Amazônia, Manaus, Brasil
}

\author{
Rosa Sá Gomes Hutchings ${ }^{1}$, Maria Anice Mureb Sallum², Ruth Leila Menezes Ferreira ${ }^{1}$ \\ $\&$ Roger William Hutchings ${ }^{3}$
}

\begin{abstract}
${ }^{1}$ Coordenação de Pesquisas em Entomologia, Instituto Nacional de Pesquisas da Amazônia, C.P. 478, 69011-970 Manaus-AM, Brasil. rsghutch@inpa.gov.br

${ }^{2}$ Departamento de Epidemiologia, Faculdade de Saúde Pública, Universidade de São Paulo, Av. Dr. Arnaldo 715, 01246-904 São Paulo-SP, Brasil. ${ }^{3}$ Coordenação de Pesquisas em Ecologia, Instituto Nacional de Pesquisas da Amazônia, C.P. 478, 69011-970 Manaus-AM, Brasil.
\end{abstract}

\begin{abstract}
The Nelson L. Cerqueira Mosquito Collection (Diptera, Culicidae) in the Invertebrate Collection of the Instituto Nacional de Pesquisas da Amazônia, Manaus, Brazil. The discovery of mosquito specimens that belonged to Nelson L. Cerqueira, which are being deposited in the Instituto Nacional de Pesquisas da Amazônia Invertebrate Collection, is reported. The collection contains 2,046 adult specimens and 387 slide preparations representing 261 species, 22 genera, including 51 paratypes of 34 species. More than $90 \%$ of the specimens were collected in Brazil of which half are from the State of Amazonas. A list of the species represented in this collection is provided indicating the number of specimens for each type of preparation and the collecting localities. The type specimens are also listed including their label data and other pertinent information.
\end{abstract}

Keywords. Brazil; Collection; Culicidae; Diptera; Nelson Cerqueira.

Resumo. O acervo de mosquitos (Diptera, Culicidae) de Nelson L. Cerqueira na Coleção de Invertebrados do Instituto Nacional de Pesquisas da Amazônia, Manaus, Brasil. Registra-se a descoberta de espécimes de mosquitos que pertenciam a Nelson L. Cerqueira, e estão sendo depositados na coleção de Invertebrados do Instituto Nacional de Pesquisas da Amazônia. A coleção contém 2.046 espécimes adultos e 387 lâminas representando 261 espécies, 22 gêneros, incluindo 51 parátipos de 34 espécies. Mais de $90 \%$ dos espécimes foram coletados no Brasil dos quais metade são do Estado do Amazonas. As espécies representadas neste acervo são listadas indicando o número de espécimes para cada tipo de preparação e as localidades de coleta. O material tipo também é listado, incluindo os dados dos rótulos de identificação e de procedência, bem como outras informações pertinentes.

Palavras-Chave. Brasil; Coleção; Culicidae; Diptera; Nelson Cerqueira.

A coleção de Culicídeos do Instituto Nacional de Pesquisas da Amazônia (INPA) foi iniciada no ano de 1954 com a transferência do Dr. Nelson Leandro Cerqueira, do Ministério da Saúde, para o quadro de pesquisadores do INPA, onde permaneceu até o seu falecimento em 1969. Esta coleção de mosquitos foi formada pelo material coletado por funcionários do INPA, chefiados por N. L. Cerqueira, incluindo indivíduos provenientes dos arredores de Manaus e de outras regiões da Amazônia. Durante este período, N. L. Cerqueira publicou vários artigos sobre a fauna de mosquitos da região (Cerqueira 1957, 1961b; Lane \& Cerqueira 1957, 1958), bem como da distribuição geográfica (Cerqueira 1961a) e a descrição de espécies novas (Cerqueira 1960a, b;1961c). Seus trabalhos representam contribuição de elevado valor para o conhecimento da fauna de mosquitos da região Amazônica.

Recentemente, chegou a nosso conhecimento a existência de material utilizado por N. L. Cerqueira que não está depositado em nenhuma coleção institucional. Aparentemente, este material proveniente de diversas localidades do Brasil bem como de outros países do continente americano, fazia parte do acervo que estava sendo utilizado por Cerqueira na ocasião da sua morte. Parte do material, que inclui adultos e lâminas (genitália, exúvias de larva e pupa), foi coletada pelo extinto Serviço Nacional de Febre Amarela do Ministério da Educação e Saúde Pública (S.F.A.M.E.S. Bras.). Também fazem parte desse material alguns exemplares-tipo, principalmente de espécies descritas por ele.

O objetivo deste trabalho é de tornar público este acervo deixado por N. L. Cerqueira e de fornecer a relação do material que está sendo catalogado e depositado na Coleção de Invertebrados do Instituto Nacional de Pesquisas da Amazônia (INPA) em Manaus.

Fazem parte do acervo, espécimes adultos, machos e fêmeas, preservados a seco e montados em alfinetes entomológicos. Há, também, espécimes imaturos, exúvias, e genitália masculina montadas em bálsamo do Canadá entre lâmina e lamínula. Para auxiliar na organização e catalogação dos espécimes, os mesmos foram numerados seqüencialmente (N. ${ }^{\circ}$ RSGH). Baseado nesta numeração, foi criado um banco de dados com as características dos espécimes (sexo, estágio, estado de conservação) incluindo as informações contidas nas etiquetas (localidade de coleta, coletor, data da coleta, número de série, identificação) junto com outras informações 
disponíveis nas mesmas. Quando necessário, os dados colhidos das etiquetas foram verificados e complementados com informações constantes no livro de entradas de artrópodos (do antigo setor de Zoologia do INPA) que foi utilizado na época para levar um controle dos números de coleta (séries) designados aos espécimes. Quando possível, as identificações constantes nas etiquetas dos espécimes foram atualizadas seguindo Guimarães (1997) e Reinert (2000).

Utilizando este banco de dados foi produzido inventário do acervo apresentado na forma de tabela com os espécimes listados em ordem alfabética por gênero e espécie acompanhado por uma listagem dos tipos em ordem alfabética por espécie. Os adultos e as lâminas muito danificados não foram incluídos na coleção. Estas informações foram organizadas em formato similar ao apresentado por Harbach et al. (1990) e Peyton et al. (1999). No texto e na tabela foram utilizadas as abreviaturas dos gêneros e subgêneros propostas por Reinert (2001).

O acervo de N. L. Cerqueira é formado por 2046 espécimes adultos e 387 lâminas de 261 espécies distribuídas em 22 gêneros. Infelizmente o estado de conservação da grande maioria do material é precário e muitos espécimes estão contaminados por fungos e outros estão com partes do corpo danificadas. O inventário geral do conteúdo da coleção, organizado por espécie, indicando o número de adultos e lâminas e sua distribuição geográfica é apresentado na Tabela I. A maioria (90\%) dos espécimes foi coletada no Brasil, seguida por Bolívia (6\%) e Peru ( $<2 \%)$. Os outros países (Argentina, Colômbia, El Salvador, Paraguai, Estados Unidos da América e Venezuela) juntos, representam menos de $2 \%$. O estado do Amazonas é o melhor representado do Brasil com 50\% do material ( $44 \%$ de todo o acervo). Todas as lâminas são de indivíduos coletados no Estado do Amazonas.

Incluídos no acervo, estão 51 parátipos (adultos) de 34 espécies, a lâmina de genitália do holótipo de Ps. (Jan.) amazonica Cerqueira, 1960 e a lâmina de genitália do holótipo de Hg. (Hag.) baresi Cerqueira,1960. Dentre o material montado em lâmina, também, encontra-se uma genitália do parátipo de Hg.(Hag.) baresi Cerqueira, 1960 e duas lâminas de exúvias de larva e pupa (p.1.) dos parátipos de Ps. (Jan.) amazonica Cerqueira,1960, respectivamente dos exemplares que estão depositados na Coleção de Invertebrados do INPA.

Relação do material tipo do acervo de N. L. Cerqueira. As espécies estão relacionadas em ordem alfabética, incluindo as seguintes informações: o nome da espécie, autor(es) e ano de publicação, classificação genérica e subgenérica atual, tipo, sexo, estágio (adulto, genitália, exúvia), dados das etiquetas, e observações dos autores que estão entre chaves \{\} . As seguintes convenções foram utilizadas no texto para representar as respectivas coleções entomológicas: IOC, Instituto Oswaldo Cruz, FIOCRUZ, Rio de Janeiro; FSP-USP, Faculdade de Saúde Pública, Universidade de São Paulo, São Paulo; INPA, Instituto Nacional de Pesquisas da Amazônia, Manaus; CPqRR, Centro de Pesquisas René Rachou, FIOCRUZ, Belo Horizonte. aenigmaticus (Cerqueira \& Costa,1946). Ochlerotatus (Och.).

Parátipo, macho, adulto, de Aedes (Ochlerotatus) aenigmaticus. Fazenda Paraíso, MT, BR, Shannon, R.C. coll., jan./1938, S.F.A. M.E.S. Bras., \{lâmina\#\} 1764T, $\left\{\mathrm{N} .{ }^{\circ} \mathrm{RSGH}\right\} 0097$.

\{Trata-se de um dos 5 parátipos machos que, segundo Cerqueira \& Costa (1946), seriam depositados no IOC, na coleção do Laboratório do Serviço de Estudos e Pesquisas sobre a Febre Amarela e no United States National Museum. O holótipo macho, que deveria estar depositado no coleção do IOC, conforme foi especificado na descrição original (Belkin et al. 1971; Marchon Silva et al. 1996), está no CPqRR (Xavier 1973). A lâmina 1764T não foi encontrada no acervo de Cerqueira\}.

airosai Lane \& Cerqueira,1942. Wyeomyia.

Parátipo, macho, adulto, de Wyeomyia (Dendromyia) airosai. Rio de Janeiro, RJ, BR, Whitman, L. coll., S.F.A. M.E.S. Bras., \{série\#\}W601, \{N. ${ }^{\circ}$ RSGH $\} 0212$.

\{Trata-se de um dos 6 parátipos fêmeas que, segundo Lane \& Cerqueira (1942), seriam depositados na Seção de Entomologia do Serviço de Estudos e Pesquisas sobre a Febre Amarela. O holótipo macho e o alótipo fêmea estão depositados no IOC (Belkin et al. 1971; Marchon Silva et al. 1996)\}.

Parátipo, fêmea, adulto, de Wyeomyia (Dendromyia) airosai. Santa Tereza, ES, BR, Whitman, L. coll., mai./ 1940, \{série\#\}L307, \{ $\left.\mathrm{N}^{\circ} \mathrm{RSGH}\right\} 0229$.

\{Trata-se de um dos 6 parátipos fêmeas que, segundo Lane \& Cerqueira (1942), seriam depositados na Seção de Entomologia do Serviço de Estudos e Pesquisas sobre a Febre Amarela\}.

alani Lane \& Cerqueira,1957. Wyeomyia (Ant.).

Parátipo, fêmea, adulto, de Wyeomyia (Antunesmyia) rooti Lane \& Cerqueira,1942. Mangaratiba, RJ, BR, S.F.A. M.E.S. Bras., $\left\{\right.$ N. ${ }^{\circ}$ RSGH $\} 0199$.

\{Trata-se de um dos 14 parátipos fêmeas que, segundo Lane \& Cerqueira (1942), seriam depositados na Seção de Entomologia do Serviço de Estudos e Pesquisas sobre a Febre Amarela. O holótipo macho e o alótipo fêmea estão depositados no IOC (Belkin et al. 1971; Marchon Silva et al. 1996)\}.

amazonica Cerqueira,1960. Psorophora (Jan.).

Genitália (holótipo) de Psorophora (Janthinosoma) amazonica. Igarapé do Tarumã, Manaus, AM, BR, Elias, C. coll.,16/jun./1956. \{série\#\}1443-60, \{lâmina \#\}386.

\{Segundo Belkin et al. (1971), esta lâmina de genitália (386) estava perdida pois não foi encontrada junto com o holótipo macho, e com a lâmina 342 p.l. (exúvias de larva e pupa), que estão depositados na FSP-USP \}.

Exúvias de larva e pupa do parátipo. Igarapé do Tarumã, Manaus, AM, BR, Elias, C. coll., 18/jun./1956, \{série\#\} 1443-40, Cerqueira, N. Det.1960., \{lâmina\#\}340. \{Esta lâmina de exúvias de larva e pupa (340 p.l.) é do parátipo macho que está depositado na Coleção de 
Invertebrados do INPA sob número Dipt.036\}.

Exúvias de larva e pupa do parátipo. Igarapé do Tarumã, Manaus, AM, BR, Elias, C. coll., 18/jun./1956. \{série\#\} 1443-33, Cerqueira, N. Det.1960., \{lâmina\#\}345. \{Esta lâmina de exúvias de larva e pupa (345 p.l.) é do parátipo fêmea que está depositado na Coleção de Invertebrados do INPA sob número Dipt.035\}.

antunesi Lane \& Guimarães, 1937. Wyeomyia (Pho.).

Síntipo fềmea adulto. C. Jordão, SP, BR, Lane, J. coll., S.F.A. M.E.S. Bras., \{série\#\} 372, \{N. ${ }^{\circ}$ RSGH $\} 0265$.

\{Trata-se de um dos 157 síntipos fêmeas que, segundo Lane \& Guimarães (1937), foram estudados como parte da descrição original. Os outros síntipos machos, fêmeas e larvas de Wy. (Pho.) antunesi estão depositados na FSP-USP e ainda falta designar o lectótipo para esta espécie (Belkin et al. 1971)\}.

baresi Cerqueira, 1960. Haemagogus (Hag.).

Genitália (holótipo). Igarapé do Tarumã, Manaus, Am, BR, Elias et al., 6/dez./1959, \{série\#\} 1607-3, \{lâmina\#\}332. \{Esta lâmina de genitália (332 g.) do holótipo foi considerada perdida por Belkin et al. (1971). O holótipo macho, e a respectiva lâmina de exúvia de pupa 316 p., estão depositados na FSP-USP \}.

Genitália (parátipo). Ponta Pelada, Manaus, AM, BR, Elias, C. \& Carlos Al. coll., 28/jan./1959, \{série\#\}1826-6, Cerqueira, N., det.1960., \{lâmina\#\}333.

\{Esta lâmina de genitália (333 g.) é do parátipo macho que está depositado na Coleção de Invertebrados do INPA sob número Dipt.034\}.

bonnei (Lane \& Cerqueira,1942). Wyeomyia (Pho.).

Parátipo, fêmea, adulto, de Phoniomyia bonnei. Rio de Janeiro, DF, BR, Whitman, L. coll., 1940, S.F.A. M.E.S. Bras., \{lâmina\#\} 1435 P.L., \{série\#\}V3, \{N. ${ }^{\circ}$ RSGH\} 0232. \{Trata-se de um dos 3 parátipos fêmeas que, segundo Lane \& Cerqueira (1942), seriam depositados na Seção de Entomologia do Serviço de Estudos e Pesquisas sobre a Febre Amarela. O holótipo macho e o alótipo fêmea estão depositados no IOC (Belkin et al. 1971; Marchon Silva et al. 1996). A lâmina 1435 p.l. não foi encontrada no acervo de Cerqueira\}.

cesari Del Ponte \& Cerqueira,1938. Wyeomyia.

Parátipo, fêmea, adulto, de Wyeomyia (Wyeomyia) cesari. Cuiabá, MT, BR, fev./1935, S.F.A. M.E.S. Bras., \{série\#\}\}3413, \{N. ${ }^{\circ}$ RSGH $\} 0278$.

\{Trata-se de um dos 5 parátipos fêmeas que, segundo Del Ponte \& Cerqueira (1938), seriam depositados na coleção do Laboratório do Serviço de Febre Amarela. O holótipo fêmea está depositado no IOC (Belkin et al. 1971; Marchon Silva et al. 1996)\}.

Parátipo, fêmea, adulto, de Wyeomyia (Wyeomyia) cesari. Cuiabá, MT, BR, fev./1935, S.F.A. M.E.S. Bras., \{série\#\} $3411,\left\{\mathrm{~N} .{ }^{\circ} \mathrm{RSGH}\right\} 0279$.

\{Trata-se de um dos 5 parátipos fêmeas que, segundo Del Ponte \& Cerqueira (1938), seriam depositados na coleção do Laboratório do Serviço de Febre Amarela\}. Parátipo, fêmea, adulto, de Wyeomyia (Wyeomyia) cesari.
Cuiabá, MT, BR, fev./1935, S.F.A. M.E.S. Bras., \{série\#\}3412, \{N. ${ }^{\circ}$ RSGH $\} 0280$.

\{Trata-se de um dos 5 parátipos fêmeas que, segundo Del Ponte \& Cerqueira (1938), seriam depositados na coleção do Laboratório do Serviço de Febre Amarela\} .

circumflava Cerqueira, 1943. Psorophora (Jan.).

Parátipo, fêmea, adulto. Axiamas, Caupolican, La Paz, Bolívia, S.F.A. M.E.S. \{série\#\}26700, \{N. ${ }^{\circ}$ RSGH $\} 0714$. \{Trata-se de um dos 7 parátipos fêmeas que, segundo Cerqueira (1943), seriam depositados na coleção do Laboratório do Serviço de Estudos e Pesquisas sobre a Febre Amarela. O holótipo fêmea encontra-se depositado no IOC (Marchon Silva et al. 1996)\}

Parátipo, fêmea, adulto. Axiamas, Caupolican, La Paz, Bolívia, S.F.A. M.E.S. \{série\# $\} 26700,\left\{N^{\circ}{ }^{\circ}\right.$ RSGH $\} 0715$. \{Trata-se de um dos 7 parátipos fêmeas que, segundo Cerqueira (1943), seriam depositados na coleção do Laboratório do Serviço de Estudos e Pesquisas sobre a Febre Amarela\}.

davisi (Lane \& Cerqueira,1942). Wyeomyia (Pho.).

Parátipo, macho, adulto, de Phoniomyia davisi. Mangaratiba, RJ, BR, Shannon, R.C. coll., mai./1938, S.F.A. M.E.S. Bras., $\left\{\right.$ N. ${ }^{\circ}$ RSGH $\} 0264$.

\{Trata-se de um dos 20 parátipos machos que, segundo Lane \& Cerqueira (1942), seriam depositados na Seção de Entomologia do Serviço de Estudos e Pesquisas sobre a Febre Amarela. O holótipo macho e o alótipo fêmea estão depositados no IOC (Belkin et al. 1971; Marchon Silva et al. 1996)\}

Parátipo, macho, adulto, de Phoniomyia davisi. Mangaratiba, RJ, BR, Shannon, R.C. coll., mai./1938, S.F.A. M.E.S. Bras., $\left\{\right.$ N. ${ }^{\circ}$ RSGH $\} 0267$.

\{Trata-se de um dos 20 parátipos machos que, segundo Lane \& Cerqueira (1942), seriam depositados na Seção de Entomologia do Serviço de Estudos e Pesquisas sobre a Febre Amarela\}.

Parátipo, macho, adulto, de Phoniomyia davisi. Teresópolis, RJ, BR, mai./1938, S.F.A. M.E.S. Bras., \{N. ${ }^{\circ}$ RSGH 30270.

\{Trata-se de um dos 20 parátipos machos que, segundo Lane \& Cerqueira (1942), seriam depositados na Seção de Entomologia do Serviço de Estudos e Pesquisas sobre a Febre Amarela\}.

Parátipo, fêmea, adulto, de Phoniomyia davisi. Mangaratiba, RJ, BR, set./1938, S.F.A. M.E.S. Bras., \{N. ${ }^{\circ}$ RSGH $\{0266$.

\{Trata-se de um dos 62 parátipos fêmeas que, segundo Lane \& Cerqueira (1942), seriam depositados na Seção de Entomologia do Serviço de Estudos e Pesquisas sobre a Febre Amarela\}.

dimidiata Cerqueira,1943. Psorophora (Gra.)

Parátipo, macho, adulto. Chilon Valegrande, Santa Cruz, Bolívia, mar./1940, S.F.A. M.E.S. Bras., \{série\#\}21840, $\left\{\mathrm{N}^{\circ}{ }^{\circ} \mathrm{RSGH}\right\} 0276$.

\{Trata-se de um dos 5 parátipos machos que, segundo Cerqueira (1943), seriam depositados na coleção do 
Laboratório do Serviço de Estudos e Pesquisas sobre a Febre Amarela. O holótipo macho e o alótipo fêmea encontram-se depositados no IOC (Belkin et al. 1971; Marchon Silva et al. 1996)\}.

Parátipo, macho, adulto. Largelinas, Santa Cruz, Bolívia, mar./1940, S.F.A. M.E.S. Bras., \{N. ${ }^{\circ}$ RSGH 0277.

\{Trata-se de um dos 5 parátipos machos que, segundo Cerqueira (1943), seriam depositados na coleção do Laboratório do Serviço de Estudos e Pesquisas sobre a Febre Amarela\}.

edwardsi (Lane \& Cerqueira,1942). Wyeomyia (Pho.).

Paralectótipo, macho, adulto, de Phoniomyia edwardsi. Rio de Janeiro, DF, BR, Whitman, L. coll., 1940, S.F.A. M.E.S. Bras., \{lâmina \#\} 2506 p.l., \{série\#\}W591, \{N. ${ }^{\circ}$ RSGH $\} 0268$.

\{Trata-se de um dos 9 paralectótipos machos que, segundo Lane \& Cerqueira (1942), seriam depositados na Seção de Entomologia do Serviço de Estudos e Pesquisas sobre a Febre Amarela. O lectótipo macho está depositado no USNM e um paralectótipo fêmea, no IOC (Belkin et al. 1971; Marchon Silva et al. 1996). A lâmina 2506 p.l. não foi encontrada no acervo de Cerqueira\}.

Paralectótipo, fêmea, adulto, de Phoniomyia edwardsi. Rio de Janeiro, RJ, BR, Whitman, L. coll., 1940, S.F.A. M.E.S. Bras., \{lâmina \#\} 2502 p.1., \{série\#\}W565, $\left\{\mathrm{N}^{\circ}{ }^{\circ}\right.$ RSGH $\} 0269$.

\{Trata-se de um dos 26 paralectótipos fêmeas que, segundo Lane \& Cerqueira (1942), seriam depositados na Seção de Entomologia do Serviço de Estudos e Pesquisas sobre a Febre Amarela. A lâmina 2502 p.l. não foi encontrada no acervo de Cerqueira .

flabellata (Lane \& Cerqueira,1942). Wyeomyia (Pho.).

Parátipo, macho, adulto, de Phoniomyia flabellata. Muriqueira, BA, BR, 26/abr./1929, slide Sab.2 / V2, ex. bromélia, rock found lab. Shannon, S.F.A. M.E.S. Bras., $\left\{\right.$ N. ${ }^{\circ}$ RSGH $\} 0231$.

\{Trata-se do único parátipo macho que, segundo Lane \& Cerqueira (1942), seria depositado na Seção de Entomologia do Serviço de Estudos e Pesquisas sobre a Febre Amarela. O holótipo macho e o alótipo fêmea estão depositados no IOC (Belkin et al. 1971; Marchon Silva et al. 1996). A lâmina Sab.2 / V2 não foi encontrada no acervo de Cerqueira\}.

Parátipo, fêmea, adulto, de Phoniomyia flabellata Lane \& Cerqueira,1942. BA, BR, \{série\#\}218, S.F.A. M.E.S. Bras., $\left\{\right.$ N. ${ }^{\circ}$ RSGH $\} 0230$.

\{Trata-se de um dos 3 parátipos fêmeas que, segundo Lane \& Cerqueira (1942), seriam depositados na Seção de Entomologia do Serviço de Estudos e Pesquisas sobre a Febre Amarela\}.

flui Bonne-Wepster \& Bonne,1919. Wyeomyia .

Parátipo, fêmea, adulto, de Wyeomyia (Dendromyia) kerri Del Ponte \& Cerqueira, 1938. Cuiabá, MT, BR, jun./ 1935, S.F.A. M.E.S. Bras., \{série\#\} 4729, \{N. ${ }^{\circ}$ RSGH $\} 0202$.
\{Trata-se de um dos 10 parátipos fêmeas que, segundo Del Ponte \& Cerqueira (1938), seriam depositados na coleção do Laboratório do Serviço de Febre Amarela. O holótipo fêmea de Wy. kerri está depositado no IOC (Belkin et al. 1971; Marchon Silva et al. 1996)\}.

Parátipo, fêmea, adulto, de Wyeomyia (Dendromyia) kerri. Cuiabá, MT, BR, fev./1935, S.F.A. M.E.S. Bras., \{série\#\}3418, \{N. $\left.{ }^{\circ} \mathrm{RSGH}\right\} 1137$.

\{Trata-se de um dos 10 parátipos fêmeas que, segundo Del Ponte \& Cerqueira (1938), seriam depositados na coleção do Laboratório do Serviço de Febre Amarela\}.

howardi Lane \& Cerqueira,1942. Wyeomyia.

Parátipo, macho, adulto, de Wyeomyia (Dendromyia) howardi. Muriqueira, BA, BR, 26/abr./1929, rock found, Lab. Shannon, Ex: Bromélia, \{N. ${ }^{\circ}$ RSGH 0274.

\{Trata-se de um dos 2 parátipos machos que, segundo Lane \& Cerqueira (1942), seriam depositados na Seção de Entomologia do Serviço de Estudos e Pesquisas sobre a Febre Amarela. O holótipo macho e o alótipo fêmea estão depositados no IOC (Belkin et al. 1971; Marchon Silva et al. 1996)\}.

Parátipo, fêmea, adulto, de Wyeomyia (Dendromyia) howardi. Muriqueira, BA, BR, 26/abr./1929, Lab.Shannon, Ex: Bromélia, \{N. ${ }^{\circ}$ RSGH $\} 0275$.

\{Trata-se de um dos 4 parátipos fêmeas que, segundo Lane \& Cerqueira (1942), seriam depositados na Seção de Entomologia do Serviço de Estudos e Pesquisas sobre a Febre Amarela\}.

knabi Lane \& Cerqueira,1942. Wyeomyia.

Parátipo, fêmea, adulto, de Wyeomyia (Dendromyia) knabi. Rio de Janeiro, RJ, BR, 01/mar./1938, Shannon, R.C. coll., S.F.A. M.E.S. Bras., $\left\{\right.$ N. $\left.{ }^{\circ} \mathrm{RSGH}\right\} 0228$.

\{Trata-se de um dos 20 parátipos fêmeas que, segundo Lane \& Cerqueira (1942), seriam depositados na Seção de Entomologia do Serviço de Estudos e Pesquisas sobre a Febre Amarela. O holótipo macho e o alótipo fêmea estão depositados no IOC (Belkin et al. 1971; Marchon Silva et al. 1996)\}.

Parátipo, fêmea, adulto. Itaituba, Fordlandia, PA, BR, 01/ fev./1938, S.F.A. M.E.S. Bras., \{série\#\} 17839, \{N. ${ }^{\circ}$ RSGH $\} 0272$.

\{Trata-se de um dos 20 parátipos fêmeas que, segundo Lane \& Cerqueira (1942), seriam depositados na Seção de Entomologia do Serviço de Estudos e Pesquisas sobre a Febre Amarela\}.

lanei Shannon \& Cerqueira,1943. Psorophora (Jan.).

Parátipo, fêmea, adulto. Porta Chuelo, Santa Cruz, Bolívia, dez./1939, S.F.A. M.E.S. Bras., \{série\#\}21571, \{N. ${ }^{\circ}$ RSGH 30592.

\{Trata-se de um dos 134 parátipos fêmeas que, segundo Shannon \& Cerqueira (1943), seriam depositados na Seção de Entomologia do Serviço de Estudos e Pesquisas sobre a Febre Amarela. O holótipo fêmea está depositado no IOC (Belkin et al. 1971; Marchon Silva et al. 1996)\}. 
limai Lane \& Cerqueira,1942. Wyeomyia (Wyo.).

Parátipo, fêmea, adulto. Londrina, PR, BR, nov./1936, S.F.A. M.E.S. Bras., \{série\#\} 11674, N $^{\circ}{ }^{\circ}$ RSGH $\} 0273$.

\{Trata-se de um dos 38 parátipos fêmeas que, segundo Lane \& Cerqueira (1942), seriam depositados na Seção de Entomologia do Serviço de Estudos e Pesquisas sobre a Febre Amarela. O holótipo macho e o alótipo fêmea estão depositados no IOC (Belkin et al. 1971; Marchon Silva et al. 1996)\}.

longipes (Fabricius, 1805). Johnbelkinia.

Parátipo, fêmea, adulto, de Trichoprosopon (Hyloconops) edwardsianus Lane \& Cerqueira,1942. Humaitá, AM, BR, nov./1937, S.F.A. M.E.S. Bras., \{série\#\} 16801, \{N. ${ }^{\circ}$ RSGH 30220 .

\{Trata-se de um dos 22 parátipos fêmeas que, segundo Lane \& Cerqueira (1942), seriam depositados na Seção de Entomologia do Serviço de Estudos e Pesquisas sobre a Febre Amarela. O holótipo fêmea de $T r$. edwardsianus está depositado no IOC (Belkin et al. 1971; Marchon Silva et al. 1996)\}.

obscurum Lane \& Cerqueira,1942. Trichoprosopon.

Parátipo, fêmea, adulto. Mangaratiba, RJ, BR, ago./1938, S.F.A. M.E.S. Bras., \{N. ${ }^{\circ}$ RSGH $\} 221$.

\{Trata-se de um dos 25 parátipos fêmeas que, segundo Lane \& Cerqueira (1942), seriam depositados na Seção de Entomologia do Serviço de Estudos e Pesquisas sobre a Febre Amarela. O holótipo macho e o alótipo fêmea estão depositados no IOC (Belkin et al. 1971; Marchon Silva et al. 1996)\}.

palmata (Lane \& Cerqueira,1942). Wyeomyia (Pho.).

Parátipo, fêmea, adulto, de Phoniomyia palmata. Rio de Janeiro, RJ, BR, Shannon, R.C. coll., dez./1937, S.F.A. M.E.S. Bras., $\left\{\right.$ N. ${ }^{\circ}$ RSGH $\} 263$.

\{Trata-se de um dos 9 parátipos fêmeas que, segundo Lane \& Cerqueira (1942), seriam depositados na Seção de Entomologia do Serviço de Estudos e Pesquisas sobre a Febre Amarela. O holótipo macho e o alótipo fêmea estão depositados no IOC (Belkin et al. 1971; Marchon Silva et al. 1996)\}.

personata (Lutz,1904). Onirion.

Paralectótipo, fêmea, adulto, de Wy. (Den.) personata. Cuiabá, MT, BR, fev./1935, S.F.A. M.E.S. Bras., \{série\#\}3314, \{No RSGH\} 1187.

\{Trata-se de uma das 8 fêmeas utilizadas originalmente por Lutz para descrever a espécie. Dendromyia personata foi descrita com base em $2 \mathrm{M}$ e $8 \mathrm{~F}$ de SP,BR e redescrita por Theobald em 1907 com base em 2M que recebeu de Lutz os quais estão depositados no The British Museum of Natural History (Belkin 1968). Um destes machos (que tem uma etiqueta indicando que tinha sido escolhido por Lane para ser o tipo) foi designado lectótipo em 1971 (Belkin et al. 1971). Os outros 7 paralectótipos fêmeas estão depositados na FSP-USP (Forattini et al. 1970)\}.

perventor (Cerqueira \& Costa,1946). Ochlerotatus (Och.).

Parátipo, fêmea, adulto, de Aedes (Ochlerotatus) perventor. Rio de Janeiro, DF, BR, Shannon, R.C. coll., dez./1937, S.F.A. M.E.S. Bras., \{lâmina\#\} 2982 p.1., \{N. ${ }^{\circ}$ RSGH\}0064.

\{Trata-se de um dos 11 parátipos fêmeas que, segundo Cerqueira \& Costa (1946), seriam depositados na coleção do Laboratório do Serviço de Estudos e Pesquisas sobre a Febre Amarela. O holótipo macho, o qual não foi encontrado no IOC conforme especificado na descrição original (Belkin et al. 1971; Marchon Silva et al. 1996), está depositado no CPqRR (Xavier 1973). A lâmina 2982 p.l. não foi encontrada no acervo de Cerqueira\}.

quasilongirostris (Theobald,1907). Wyeomyia (Pho.).

Parátipo, fêmea, adulto, de Phoniomyia neivai Lane \& Cerqueira, 1942. Londrina, PR, BR, fev./1937, S.F.A.M.E.S. Bras., \{série\#\} 12399, \{N. ${ }^{\circ}$ RSGH $\} 0218$. \{Trata-se de um dos 54 parátipos fêmeas que, segundo Lane \& Cerqueira (1942), seriam depositados na Seção de Entomologia do Serviço de Estudos e Pesquisas sobre a Febre Amarela. O holótipo fêmea de Phoniomyia neivai encontra-se depositado no IOC (Belkin et al. 1971; Marchon Silva et al. 1996)\}.

reversa (Lane \& Cerqueira,1942). Runchomyia (Run.).

Parátipo, fêmea, adulto, de Trichoprosopon (Hyloconops) reversus. Magé, RJ, BR, Shannon, R.C. coll., mai./1940, S.F.A. M.E.S. Bras., \{lâmina\#\} 2063 p.1., \{série\#\} RCS47, $\left\{\right.$ N. ${ }^{\circ}$ RSGH $\} 0222$.

\{Trata-se de um dos 242 parátipos fêmeas que, segundo Lane \& Cerqueira (1942), seriam depositados na Seção de Entomologia do Serviço de Estudos e Pesquisas sobre a Febre Amarela. O holótipo macho e o alótipo fêmea estão depositados no IOC (Belkin et al. 1971; Marchon Silva et al. 1996). A lâmina 2063 p.l. não foi encontrada no acervo de Cerqueira\}.

Parátipo, fêmea, adulto, de Trichoprosopon (Hyloconops) reversus. Magé, RJ, BR, Shannon, R.C. coll., jul./1940, S.F.A. M.E.S. Bras., \{lâmina \#\} 3006 p.1., \{série\#\}S196, $\left\{\right.$ N. ${ }^{\circ}$ RSGH $\} 0223$.

\{Trata-se de um dos 242 parátipos fêmeas que, segundo Lane \& Cerqueira (1942), seriam depositados na Seção de Entomologia do Serviço de Estudos e Pesquisas sobre a Febre Amarela. A lâmina 3006 p.l. não foi encontrada no acervo de Cerqueira\}.

rooti (Del Ponte,1939). Wyeomyia.

Paralectótipo, fêmea, adulto, de Wyeomyia (Dendromyia) delpontei Lane \& Cerqueira, 1942. Santa Isabel, Terc.Trav., PA, BR, mai./1937, S.F.A. M.E.S. Bras., \{série\#\} 14174, $\left\{\right.$ N. ${ }^{\circ}$ RSGH $\} 0213$.

\{Trata-se de um dos 11 paralectótipos fêmeas que, segundo Lane \& Cerqueira (1942), seriam depositados na Seção de Entomologia do Serviço de Estudos e Pesquisas sobre a Febre Amarela. O lectótipo fêmea de Wyeomyia rooti (Del Ponte,1939) (o mesmo espécime que é holótipo de delpontei Lane \& Cerqueira,1942) está depositado no IOC (Belkin et al. 1971; Marchon Silva et al. 1996)\}. 


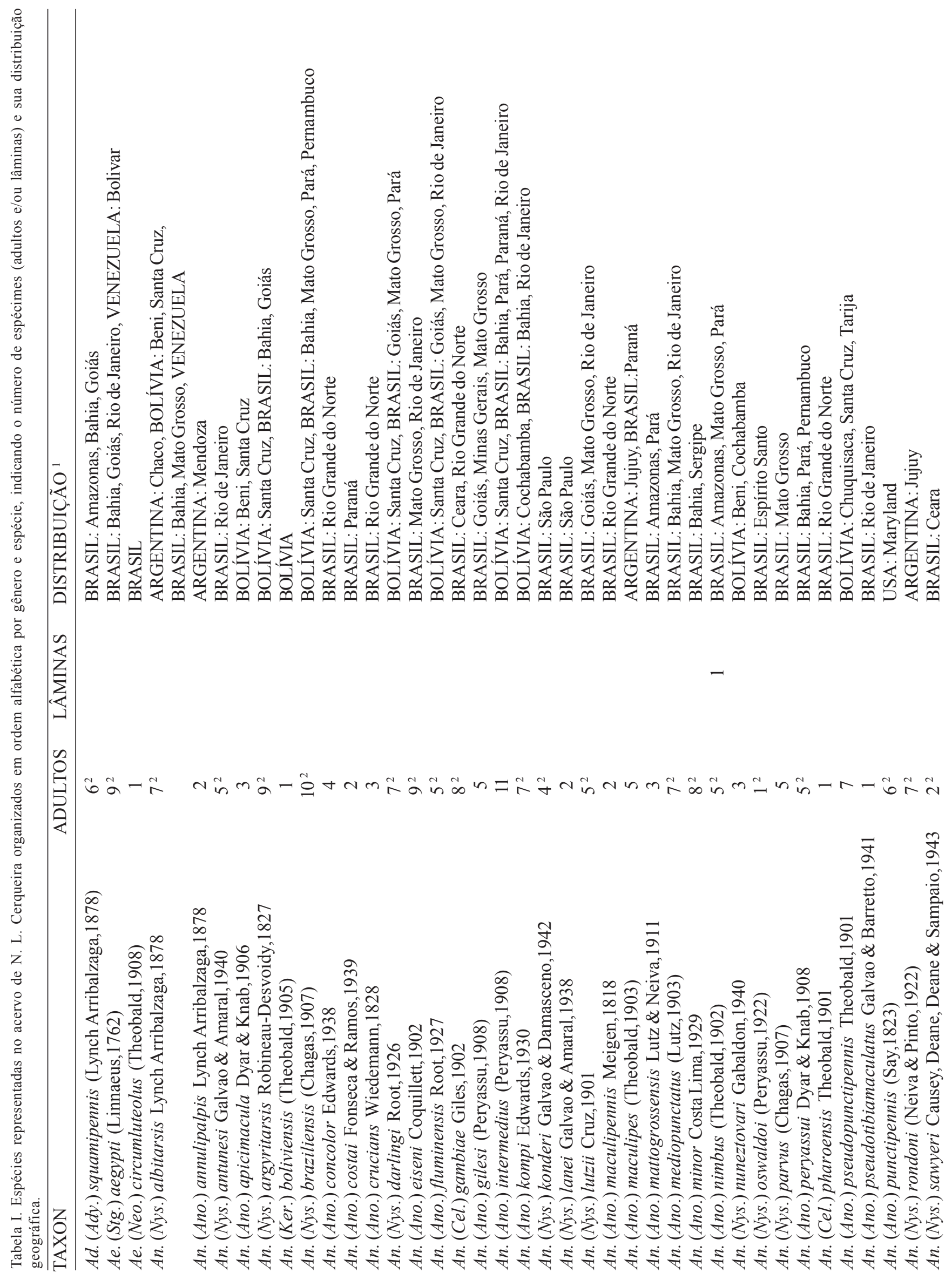









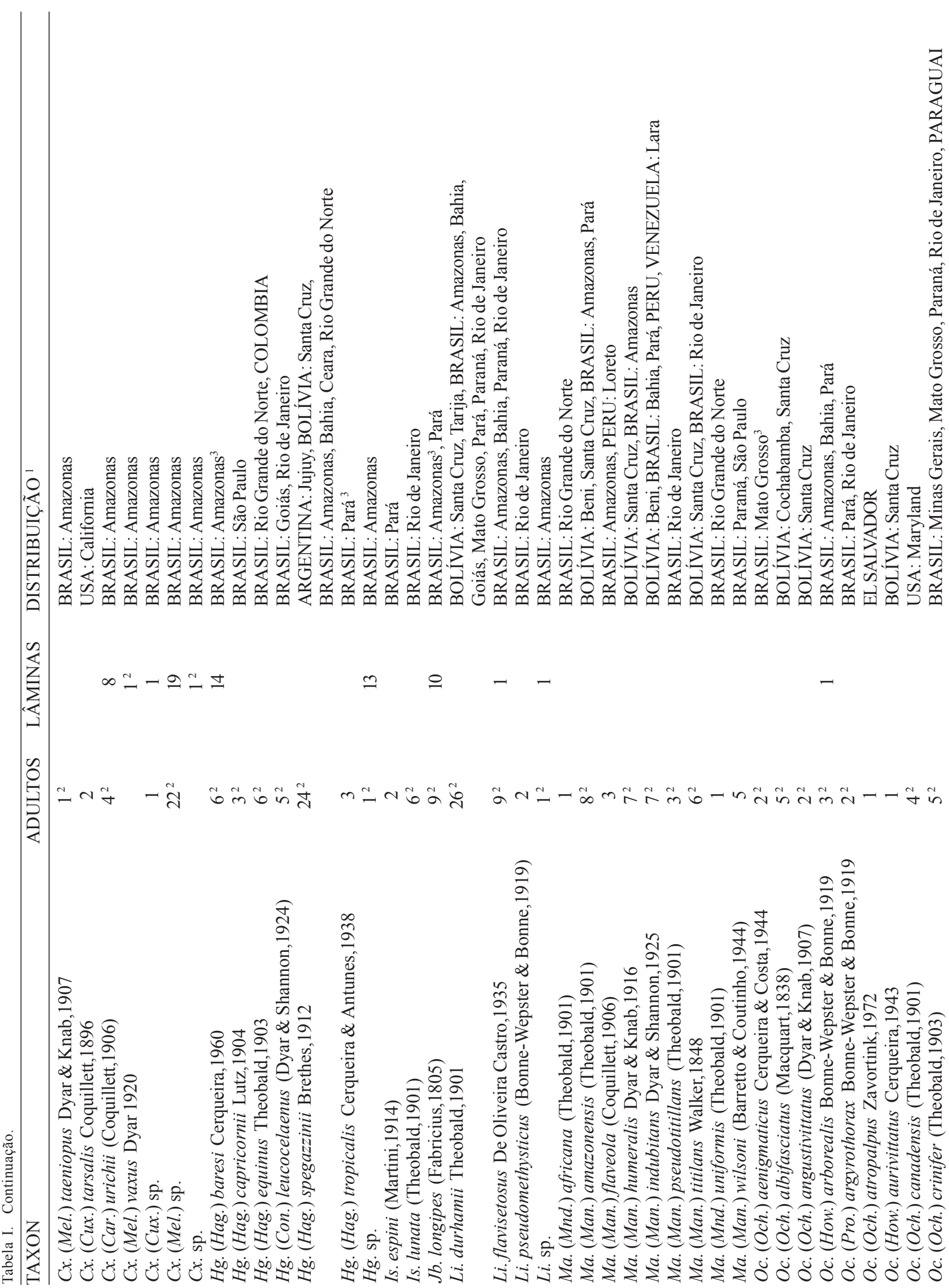




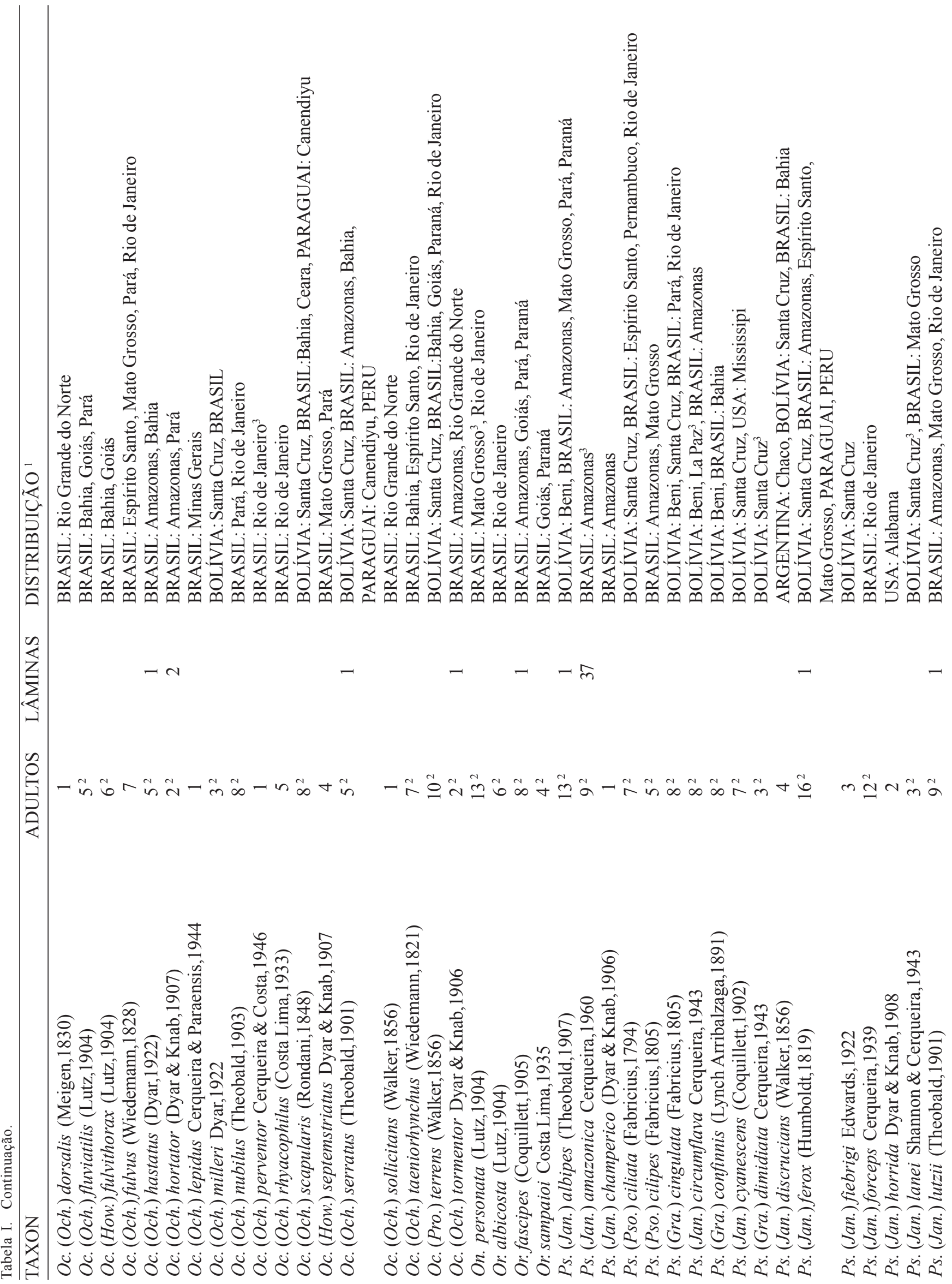




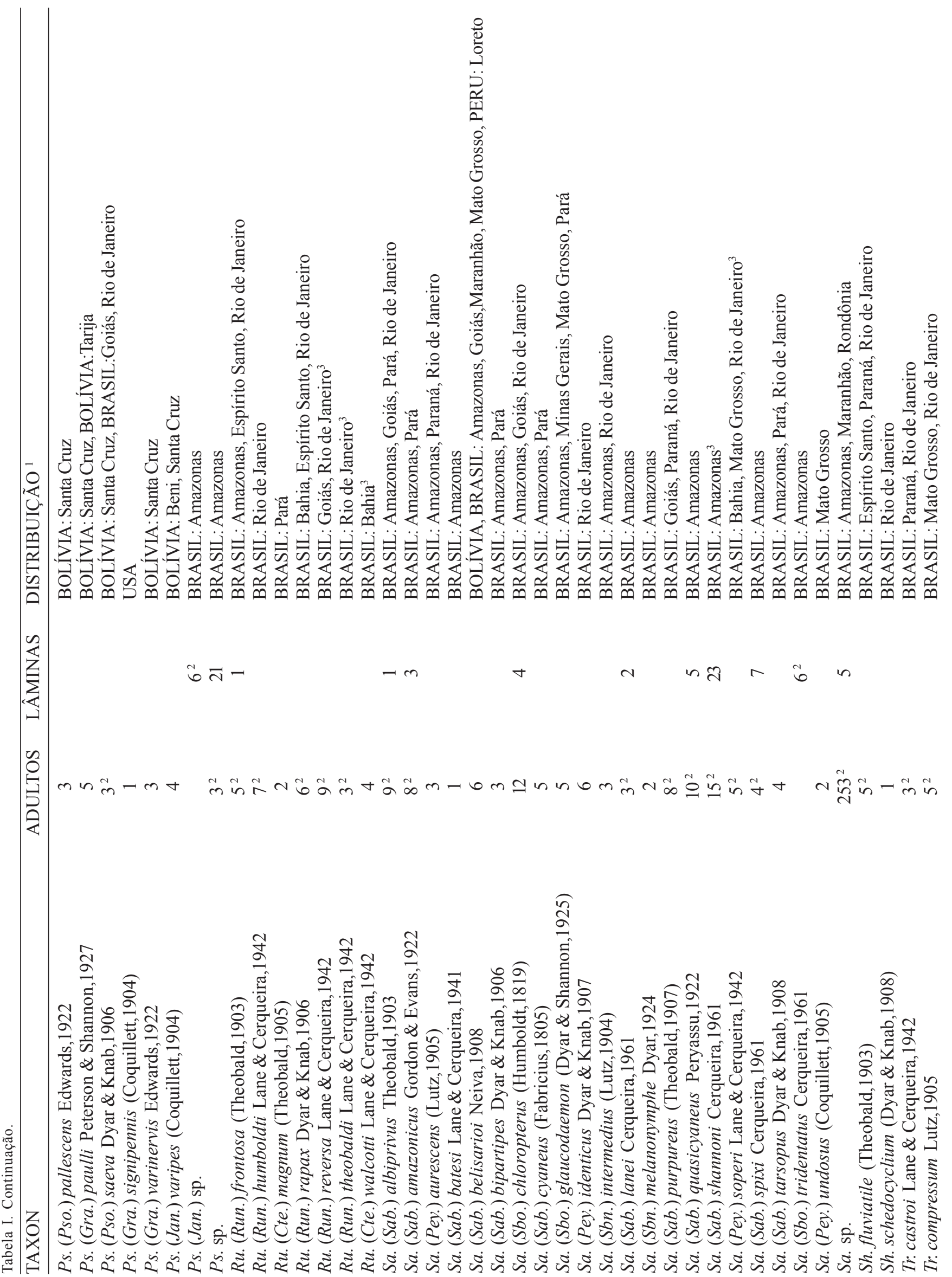









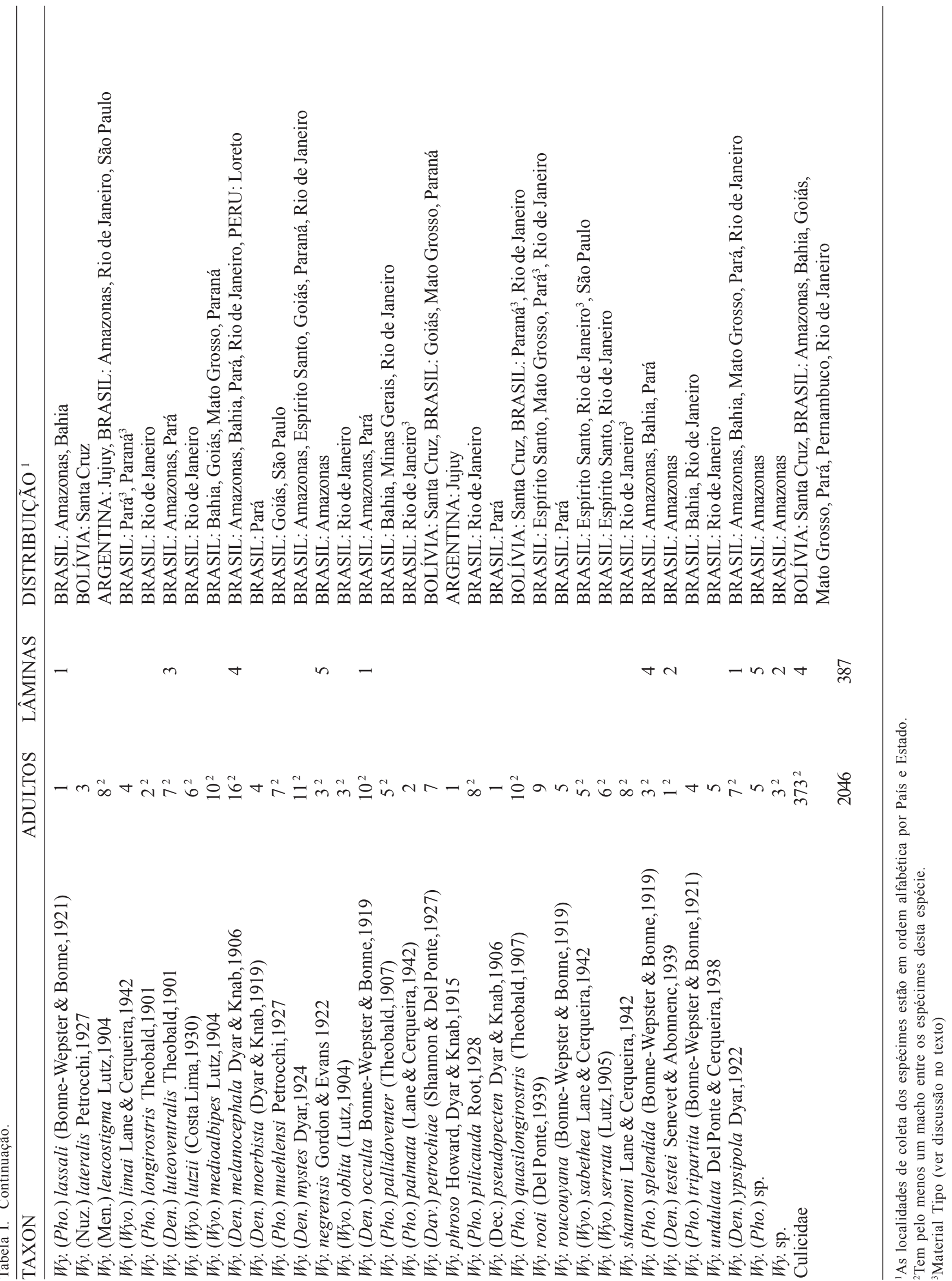


sabethea Lane \& Cerqueira,1942. Wyeomyia (Wyo.).

Parátipo, macho, adulto. Petrópolis, RJ, BR, Shannon, R.C. coll., abr./1936, S.F.A. M.E.S. Bras., $\left\{\right.$ N. ${ }^{\circ}$ RSGH $\} 0271$.

\{Trata-se de um dos 5 parátipos machos que, segundo Lane \& Cerqueira (1942), seriam depositados na Seção de Entomologia do Serviço de Estudos e Pesquisas sobre a Febre Amarela. O holótipo macho (montado em lâmina) e o alótipo fêmea estão depositados no IOC (Belkin et al. 1971; Marchon Silva et al. 1996)\}.

shannoni Cerqueira, 1961. Sabethes (Sab.).

Parátipo, fêmea, adulto. Igarapé Mariano, Manaus, AM, BR, Mozarth coll., 6/abr./1956., \{série\#\} 1357-06, \{N. ${ }^{\circ}$ RSGH 0211.

\{Trata-se de um dos 75 parátipos fêmeas que, segundo Cerqueira (1961c), seriam depositados na FSP. O holótipo macho encontra-se depositado na FSP-USP (Belkin et al. 1971)\}.

shannoni Lane \& Cerqueira,1942. Wyeomyia.

Parátipo, macho, adulto, de Wyeomyia (Dendromyia) shannoni. Teresópolis, RJ, BR, Shannon, R.C. coll., mai./ 1938, S.F.A. M.E.S. Bras., \{N. ${ }^{\circ}$ RSGH $\} 0215$.

\{Trata-se de um dos 26 parátipos machos que, segundo Lane \& Cerqueira (1942), seriam depositados na Seção de Entomologia do Serviço de Estudos e Pesquisas sobre a Febre Amarela. O holótipo macho e o alótipo fêmea estão depositados no IOC (Belkin et al. 1971; Marchon Silva et al. 1996)\}.

Parátipo, macho, adulto, de Wyeomyia (Dendromyia) shannoni. Mangaratiba, RJ, BR, Shannon, R.C. coll., S.F.A. M.E.S. Bras., \{N. ${ }^{\circ}$ RSGH $\} 0214$.

\{Trata-se de um dos 26 parátipos machos que, segundo Lane \& Cerqueira (1942), seriam depositados na Seção de Entomologia do Serviço de Estudos e Pesquisas sobre a Febre Amarela\}.

Parátipo, fêmea, adulto, de Wyeomyia (Dendromyia) shannoni. Mangaratiba, RJ, BR, ago./1938, S.F.A. M.E.S. Bras., $\left\{\right.$ N. ${ }^{\circ}$ RSGH $\} 0216$.

\{Trata-se de um dos 30 parátipos fêmeas que, segundo Lane \& Cerqueira (1942), seriam depositados na Seção de Entomologia do Serviço de Estudos e Pesquisas sobre a Febre Amarela\}.

Parátipo, fêmea, adulto de Wyeomyia (Dendromyia) shannoni. Mangaratiba, RJ, BR, ago./1938, S.F.A. M.E.S. Bras., $\left\{\right.$ N. $^{\circ}$ RSGH $\} 0217$.

\{Trata-se de um dos 30 parátipos fêmeas que, segundo Lane \& Cerqueira (1942), seriam depositados na Seção de Entomologia do Serviço de Estudos e Pesquisas sobre a Febre Amarela\} .

soaresi Lane \& Cerqueira,1942. Trichoprosopon.

Parátipo, macho, adulto. S. J. Petrópolis, 60 Bambu, ES, BR, Shannon, R.C. coll., 05/fev./1941, \{série\#\} S001, \{lâmina \#\} 2707 p., $\left\{\right.$ N. ${ }^{\circ}$ RSGH 0225.

\{Trata-se de um dos 5 parátipos machos que, segundo Lane \& Cerqueira (1942), seriam depositados na Seção de Entomologia do Serviço de Estudos e Pesquisas sobre a Febre Amarela. O holótipo macho e o alótipo fêmea estão depositados no IOC (Belkin et al. 1971; Marchon Silva et al. 1996). A lâmina 2707 p. não foi encontrada no acervo de Cerqueira\} .

soperi Lane \& Cerqueira,1942. Sabethes (Pey.).

Parátipo, macho, adulto, de Sabethes (Sabethinus) soperi. Iguassú, RJ, BR, Whitman, L. coll., ago./1940, S.F.A. M.E.S. Bras., \{série\#\}X015, \{lâmina \#\} 3027 p.1., \{N. ${ }^{\circ}$ RSGH $\} 0219$.

\{Trata-se de um dos 14 parátipos machos que, segundo Lane \& Cerqueira (1942), seriam depositados na Seção de Entomologia do Serviço de Estudos e Pesquisas sobre a Febre Amarela. O holótipo macho e o alótipo fêmea estão depositados no IOC (Belkin et al. 1971; Marchon Silva et al. 1996). A lâmina 3027 p.l. não foi encontrada no acervo de Cerqueira\}.

Parátipo, fêmea, adulto, de Sabethes (Sabethinus) soperi. Iguassú, RJ, BR, Whitman, L. coll., ago./1940, S.F.A. M.E.S. Bras., \{série\#\} X018, \{lâmina \#\} 3028 p., \{N. ${ }^{\circ}$ RSGH $\{0233$.

\{Trata-se de um dos 59 parátipos fêmeas que, segundo Lane \& Cerqueira (1942), seriam depositados na Seção de Entomologia do Serviço de Estudos e Pesquisas sobre a Febre Amarela. A lâmina 3028 p. não foi encontrada no acervo de Cerqueira\}.

theobaldi (Lane \& Cerqueira,1942). Runchomyia (Run.).

Parátipo, fêmea, adulto, de Trichoprosopon (Hyloconops) theobaldi. Magé, RJ, BR, Shannon, R.C. coll., 13/jun./ 1940, \{série\#\}RCS36, \{N. ${ }^{\circ}$ RSGH 0226.

\{Trata-se de um dos 2 parátipos fêmeas que, segundo Lane \& Cerqueira (1942), seriam depositados na Seção de Entomologia do Serviço de Estudos e Pesquisas sobre a Febre Amarela. O holótipo macho e o alótipo fêmea estão depositados no IOC (Belkin et al. 1971; Marchon Silva et al. 1996)\}.

Parátipo, macho, adulto, de Trichoprosopon (Hyloconops) theobaldi. Magé, RJ, BR, Shannon, R.C. coll., 18/jun./ 1940, S.F.A. M.E.S. Bras., \{série\#\}S098, \{N. ${ }^{\circ}$ RSGH $\} 0227$.

\{Trata-se de um dos 3 parátipos machos que, segundo Lane \& Cerqueira (1942), seriam depositados na Seção de Entomologia do Serviço de Estudos e Pesquisas sobre a Febre Amarela\}.

tropicalis Cerqueira \& Antunes, 1938. Haemagogus (Hag.).

Parátipo, macho, adulto. Curralinho, PA, BR, jan./1936, S.F.A. M.E.S. Bras., \{série\# $\} 6074,\left\{\right.$ N. ${ }^{\circ}$ RSGH $\} 0142$.

\{Trata-se de um dos 17 parátipos machos que, segundo Cerqueira \& Antunes (1938), seriam depositados no Serviço da Febre Amarela. O holótipo macho e o alótipo fêmea que seriam depositados no IOC não foram encontrados (Belkin et al. 1971; Marchon Silva et al. 1996)\}.

walcotti (Lane \& Cerqueira,1942). Runchomyia (Cte.).

Parátipo, fêmea, adulto, de Trichoprosopon (Ctenogoeldia) walcotti. Caravelas, BA, BR, jan./1931, S.F.A. M.E.S. Bras., \{série\#\} 558, \{N. ${ }^{\circ}$ RSGH $\} 0224$.

\{Trata-se de um dos 64 parátipos fêmeas que, segundo 
Lane \& Cerqueira (1942), seriam depositados na Seção de Entomologia do Serviço de Estudos e Pesquisas sobre a Febre Amarela. O holótipo fêmea encontra-se depositado no IOC (Belkin et al. 1971; Marchon Silva etal. 1996)\}.

Agradecimentos. Este trabalho foi executado utilizando recursos do Instituto Nacional de Pesquisas da Amazônia (INPA PPI 1-3580 / 2002-2003 "Biodiversidade de Insetos Amazônicos e Gerenciamento da Coleção de Insetos do INPA" coordenado pelo Dr. José Albertino Rafael). MAMS tem financiamento para pesquisa do "National Research Council", Estados Unidos da America, DAMD 17-00-2-0002.

\section{REFERÊNCIAS}

Belkin, J. N. 1968. Mosquito studies (Diptera, Culicidae). IX. The type specimens of New World mosquitoes in European museums. Contributions of the American Entomological Institute 3: 1-69.

Belkin, J. N.; R. X. Schick \& S. J. Heinemann. 1971. Mosquito studies (Diptera: Culicidae) XXV. Mosquitoes originally described from Brazil. Contributions of the American Entomological Institute 7: 1-64.

Cerqueira, N. L. \& P. C. A. Antunes. 1938. Haemagogus tropicalis, a new species from Pará, Brazil (Diptera, Culicidae). Proceedings of the Entomological Society of Washington 40: 1-9.

Cerqueira, N. L. 1943. Algumas espécies novas da Bolívia, e referência a três espécies de Haemagogus (Diptera, Culicidae). Memórias do Instituto Oswaldo Cruz 39: 1-21.

Cerqueira, N. L. \& A. F. Costa. 1946. Duas novas espécies de Aedes (Ochlerotatus) (Diptera Culicidae). Livro de homenagem a R.F. d'Almeida 11: 133-139.

Cerqueira, N. L. 1957. Descrição da larva, pupa de Aedes (Ochlerotatus) lepidus Cerqueira \& Paraense (Diptera: Culicidae, Culicini). Boletim do Museu Paraense Emilio Goeldi 3: 1-6.

Cerqueira, N. L. 1960a. Uma nova espécie de Haemagogus do Amazonas (Diptera Culicidae). Boletim do Museu Paraense Emilio Goeldi 25: $1-7$

Cerqueira, N. L. 1960b. Sôbre uma n.sp. de Janthinosoma da série Lutzi, a descrição do macho de Psorophora (J.) circumflava Cerqueira, 1943. Boletim do Museu Paraense Emilio Goeldi 26: $1-10$

Cerqueira, N. L. 1961a. Distribuição geográfica dos mosquitos da Amazônia (Diptera, Culicidae, Culicinae). Revista Brasileira de Entomologia 10: 111-168.

Cerqueira, N. L. 1961b. Sobre Trichoprosopon (R.) edwardsianum (Lane \& Cerqueira, 1942) (Diptera Culicidae). Studia Entomologica 4: 459-464.

Cerqueira, N. L. 1961c. Cinco novos Sabetinos da Amazônia (Diptera Culicidae). Revista Brasileira de Entomologia 10: 37-52.

Deane, M. P.; O. R. Causey \& L. M. Deane. 1947. Chave ilustrada para a identificação de trinta e duas espécies de Anofelinos das regiões nordestina e amazônica do Brasil, pelos caracteres da larva, com a descrição de duas larvas. Revista do Serviço Especial de Saúde Pública 1: $355-384$

Del Ponte, E. \& N. L. Cerqueira. 1938. Alguns Sabetíneos do Brasil (Diptera: Culicidae). Revista de Entomologia 8: 225-237.

Forattini, O. P.; E. X. Rabello \& M. das Dores Cotrim. 1970. Catálogo das coleções entomológicas da Faculdade de Saúde Pública da Universidade de São Paulo (1a Série) Culicidae. Revista de Saúde Pública 4 (No. Especial): 1-100.

Guimarães, J. H. 1997. Systematic Database of Diptera of the Americas South of the United States (Family Culicidae). Plêiade/Fapesp, São Paulo. 286 p.

Harbach, R. E.; T. V. Gaffigan \& J. E. Pecor. 1990. The J. Pedro Duret mosquito collection (Diptera: Culicidae). Mosquito Systematics 22: 192-195.

Lane, J. \& N. L. Cerqueira. 1942. Os Sabetíneos da América (Diptera, Culicidae). Arquivos de Zoologia do Estado de São Paulo III(IX): 473-849.

Lane, J. \& N. L. Cerqueira. 1957. The validity and change of name of two species of Wyeomyia (Diptera, Culicidae). Proceedings of the Entomological Society of Washington 59: 244.

Lane, J. \& N. L. Cerqueira. 1958. The types of Wyeomyia dyari Lane \& Cerqueira, 1942 (Diptera, Culicidae). Proceedings of the Entomological Society of Washington 60: 132.

Lane, J. \& L. R. Guimarães. 1937. Notas sobre culicideos de Campos do Jordão. Annaes Paulistas de Medicina e Cirurgia 33: 211217.

Marchon Silva, V.; R. Lourenço de Oliveira; M. D. Almeida; A. D. Silva Vasconcelos \& J. Costa. 1996. The type specimens of mosquitoes (Diptera, Culicidae) deposited in the Entomological Collection of the Instituto Oswaldo Cruz, Rio de Janeiro, Brazil. Memórias do Instituto Oswaldo Cruz 91: 471-478.

Mendonça, F. C. D. \& N. L. Cerqueira. 1947. Insects and other arthropods captured by the Brazilian Sanitary Service on landplanes or seaplanes arriving in Brazil between January 1942 and December 1945. Pan American Sanitary Bureau (Sanitary Service) January 1947: 1-9.

Peyton, E. L.; J. E. Pecor; T. V. Gaffigan; M. Trpis; L. M. Rueda \& R. C. Wilkerson. 1999. The Johns Hopkins University School of Hygiene and Public Health, Lloyd E. Rozeboom Mosquito Collection. Journal of the American Mosquito Control Association 15: 526-551.

Reinert, J. F. 2000. New classification for the composite genus Aedes (Diptera: Aedini), elevation of subgenus Ochlerotatus to generic Rank, reclassification of the other subgenera, and notes on certain subgenera and species. Journal of the American Mosquito Control Association 16: 175-188.

Reinert, J. F. 2001. Revised list of abbreviations for genera and subgenera of Culicidae (Diptera) and notes on generic and subgeneric changes. Journal of the American Mosquito Control Association 17: 51-55.

Shannon, R. C. \& N. L. Cerqueira. 1943. Psorophora lanei, um novo mosquito do Brasil e da Bolívia (Dip. Culicidae). Revista de Entomologia 14: 135-137.

Xavier, S. H. 1973. Mosquitos types of Brazil. Mosquito Systematics 5: $159-160$.

Recebido em 27.IV.2004; aceito em 02.IX.2004 